\title{
Heart transplant and oral health: the complications
}

Oral health complications after a heart transplant: a review. Br Dent J 2020; 228: 177-182.

http://dx.doi.org/10.1038/s41415-020-1244-0

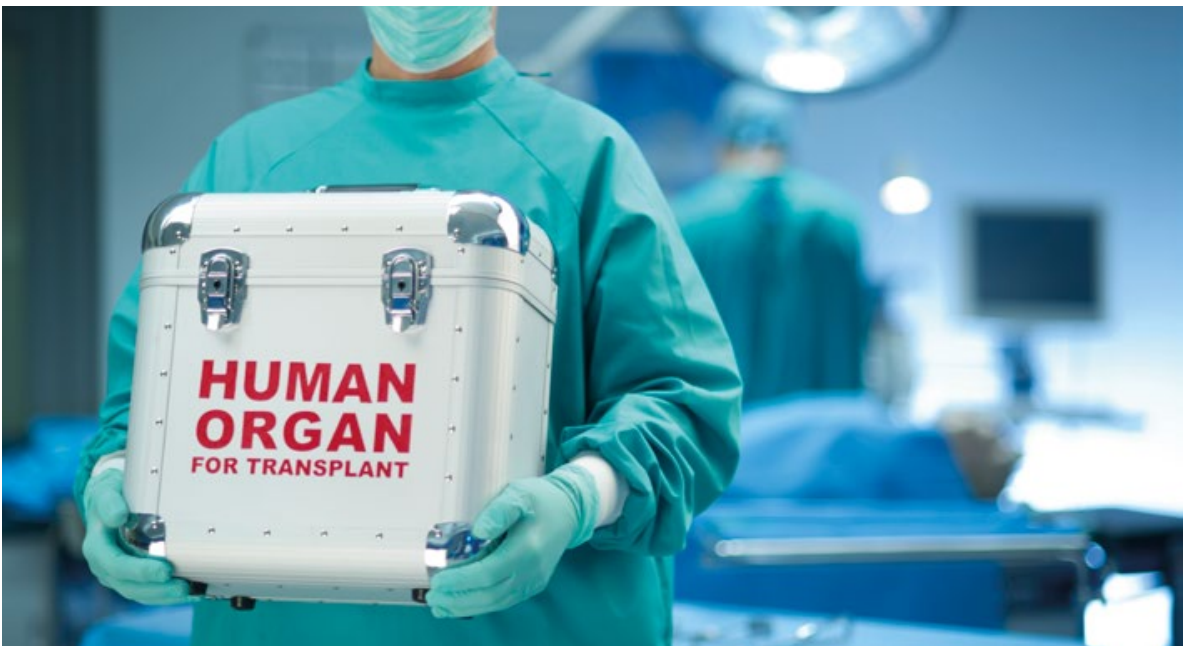

The first heart transplant in the United Kingdom was successfully performed in 1979 . Since then, the surgery now numbers 200 cases per year countrywide. Following heart transplants, patients are routinely prescribed lifelong immunosuppressants to prevent organ rejection. Many studies have established that immunosuppressants, particularly cyclosporin A (CsA), can cause patients to develop gingival hyperplasia. It begs the question - what other oral health complications could it potentially cause?

This review aimed to answer this question by looking into the current literature on oral health complications following heart transplants. The extensive PubMed literature search yielded 24 studies for this review. Among others, the complications evaluated are gingival hyperplasia, periodontal conditions, caries, Candida infection, oral malignancies and craniofacial growth.

Many have reported the occurrence of gingival hyperplasia to be between $8.3-67 \%$ among heart transplant patients who underwent CsA treatments. Some also compared the occurrence between the different types of immunosuppressants. For example, patients receiving tacrolimus treatment exhibited lower gingival hyperplasia presence (0\%), compared to those on CsA (40.7\%). On the other hand, patients undergoing a combined CsA and nifedipine treatment showed a significantly higher gingival overgrowth score $(62 \%)$ than those on a CsA treatment alone (25.8\%). In turn, patients with gingival hyperplasia manifested worse periodontal conditions. In assessing the periodontal conditions, one study reported that heart transplant patients had significantly higher scores in all periodontal indices compared to the control group. However, patients receiving periodontal treatment six months after transplantation improved their periodontal outcome 12 months after transplantation, with better gingival hyperplasia outcome 36 months after transplantation. Heart transplant patients also recorded an incidence of fungal infection between 10-25\%, with the majority scoring positive for the presence of Candida species, particularly C. albicans. When studying the incidence rate of oral cancer, a study in Canada reported that these patients were 4.3 times more likely to develop oral cancer. Interestingly, there was no difference in caries prevalence between this group and the control group. Similarly, the craniofacial growth among paediatric heart transplant patients displayed no deviation in growth pattern.

In conclusion, this review demonstrated that heart transplant patients are more likely to experience gingival hyperplasia, periodontal disease, fungal infection and oral malignancies. It accentuated the need for good oral healthcare within this group. As dental practitioners, we need to educate heart transplant patients on maintaining good oral health and treat any ensuing oral health-related problems.

By Othman Zuhir

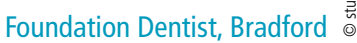

